\title{
An Intelligent System for the Diagnosis of Skin Cancer on Digital Images taken with Dermoscopy
}

\author{
Heydy Castillejos-Fernández $z^{\mathrm{a}}$, Omar López-Ortega ${ }^{\mathrm{a}}$, Félix \\ Castro-Espinoza ${ }^{a}$ and Volodymyr Ponomaryov ${ }^{b}$
}

${ }^{a}$ Universidad Autónoma del Estado de Hidalgo, Área Académica de Computación y Electrónica, Carretera Pachuca - Tulancingo km. 4.5, Mineral de la Reforma, Hidalgo, México. C. P. 42083

heydy_castillejos@uaeh.edu.mx, lopezo@uaeh.edu.mx, fcastro@uaeh.edu.mx

${ }^{\mathrm{b}}$ Instituto Politécnico Nacional, ESIME Unidad Profesional Culhuacan

Avenida Santa Ana 1000, Coyoacan, San Francisco Culhuacan,

Ciudad de México, México. C.P. 04430

vponomar@ipn.mx

\begin{abstract}
Skin cancer is a major health issue affecting a vast segment of the population regardless the skin color. This affectation can be detected using dermoscopy to determine whether the visible spots on skin are either benign or malignant tumors. In spite of the specialists' experience, skin lesions are difficult to classify, reason for which computer systems are developed to increase the effectiveness of cancer detection. Systems assisting in the detection of skin cancer process digital images to determine the occurrence of tumors by interpreting clinical parameters, relying, firstly, upon an accurate segmentation process to extract relevant features. Two of the well-known methods to analyze lesions are $A B C D$ (Asymmetry, Border, Color, Differential structures) and the 7-point check list. After clinically-relevant features are extracted, they are used to classify the presence or absence of a tumor. However, irregular and disperse lesion borders, low contrast, artifacts in images and the presence of various colors within the region of interest complicate the processing of images. In this article, we propose an intelligent system running the following method. The feature extraction stage begins with the segmentation of an image, for which we apply the Wavelet - Fuzzy C-Means algorithm. Next, specific features should be determined, among others the area and the asymmetry of the lesion. An ensemble of clusterers extracts the Red-Green-Blue values that correspond to one or more of the colors defined in the $A B C D$ guide. The feature extraction stage includes the discovery of structures that appear in the lesion according to the method known as Grey Level CoOccurrence Matrix (GLCM). Then, during the detection phase, an ensemble of classifiers determines the occurrence of a malignant tumor. Our experiments are performed on images taken from the ISIC repository. The proposed system provides a skin cancer detection performance above 88 percent, as measured by the accuracy. Details of how this performance fares when compared with other systems are also given.
\end{abstract}


Keywords: segmentation; fuzzy logic; color detection; classification

\section{Introduction}

Skin cancer is a major health issue affecting vast segments of the population regardless the skin color. Data indicate that the incidence of melanoma, which is a type of cancer that metastasizes rapidly, has increased alarmingly. It begins by modifying melanocytes (epidermal cell that produces melanin) of normal skin or moles, resulting as a dark area on the skin. This damaging process changes the normal concentration of melanin (dark-brown, black or reddish-brown substance that is natural of people's skin, hair and eyes). Because this affectation is apparent on skin, it is possible to use a non-invasive technique called dermoscopy (derma scope) to determine whether the visible spots on skin are either benign or malignant tumors.

Numerous techniques have been proposed in order to characterize and define patterns and structures of pigmented and non pigmented skin lesions. Nonetheless, skin lesions are difficult to classify, reason for which computer-based systems are developed to improve the detection of skin cancer through the extraction and interpretation of several clinical parameters. Generally, the following stages must be completed by any computerized diagnostic system:

- Pre-processing. In this stage, filters for removal artifacts are applied.

- Image segmentation. A specific region of the lesion is separated from the rest of the original digital image.

- Feature extraction. Clinically-relevant features that are defined in various guides, among others the ABCD (Asymmetry, Border, Color, Differential structures), must be extracted correctly in order to interpret the lesion. Another guideline that could be implemented is a checklist of 7 criteria that define a malignant tumor.

- Learning and diagnosis. This stage is facilitated by employing machine learning techniques, i.e. classifiers.

Thus, intelligent systems must implement an accurate image segmentation process to analyze borders, colors, and structures of a lesion. This requirement is compulsory to extract clinically relevant features of dermoscopy images. However, irregular and disperse lesion borders, low contrast, artifacts in images and variety of colors within the interest region pose a tremendous challenge in the segmentation step. After the segmentation and feature extraction processes are complete, the set of relevant features must be classified accurately to determine the presence of a malignant tumor or discard its occurrence. 
To solve these two major problems (feature extraction and classification) we propose an intelligent system for detecting whether a lesion is a benign or malignant tumor. The proposed intelligent system executes the following method. First, feature extraction is achieved by segmenting the image of the skin lesion with the Wavelet Fuzzy C Means (W-FCM) algorithm [1]. When the lesion segmentation is done, the following features are obtained: asymmetry, all the features considered in the Grey-Level Co-Occurrence Matrix (GLCM) and, as novel proposals, our method includes the extraction of the eccentricity value and the color content of the lesion. The color extraction is performed by an ensemble of clusterers that estimates the presence of one or more colors following the ABCD guide.

After the feature extraction phase is terminated, the learning phase of the intelligent system commences. We propose an ensemble of classifiers as a means to elevate the accuracy of classifying the lesion as either benign or malignant. We measure the effectiveness of the classification task by calculating values for sensibility, specificity, accuracy and the area under the ROC curve.

Our experiments were done on images taken from the ISIC repository. With the method proposed, our system provides a skin cancer detection performance ranking at the top tier, as contrasted with other systems that have been reported. The paper is organized as follows. Section 2 presents the method that covers the lesion segmentation, color and clinically-relevant features extraction, and learning. Section 3 contains detailed experimental results. A comparison of our system performance with other systems is given in Section 4. Finally, conclusions and future work are delineated.

\section{The Proposed Method}

The method that we propose is illustrated in Figure 1, where each of the stages is represented with a dash-lined rectangle. The relevant stages of the method are: Lesion segmentation and feature extraction based on W-FCM; color extraction based on an ensemble of clusterers; creation of the features vector and, finally, learning and prediction based on an ensemble of classifiers.

Each phase is explained next. 


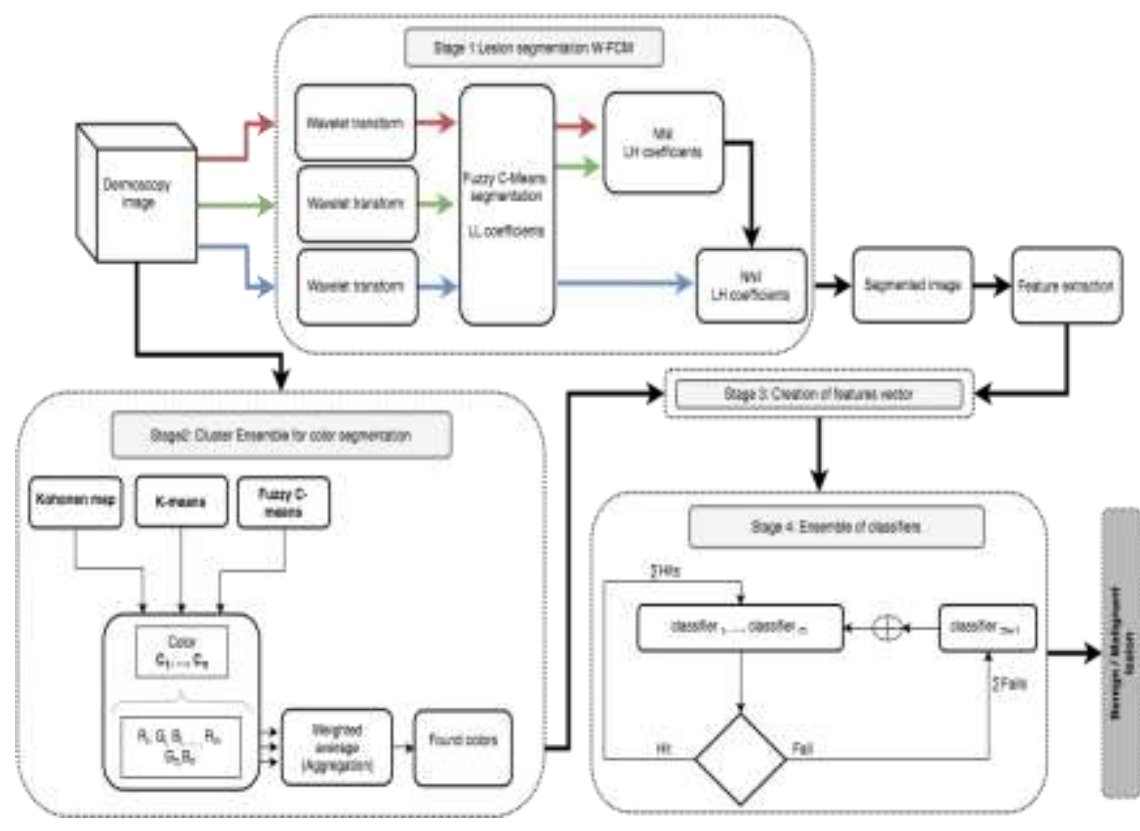

Figure 1

Block diagram of the proposed method to determine whether a lesion is a benign or malignant tumor

\subsection{Lesion Segmentation}

Before the segmentation process, we employ a framework that employs the feature extraction in Wavelet Transform (WT) space. This operation is paramount because it is possible to obtain data from the Red, Blue and Green channels of a digital image $[1,2]$. The acquisition of the three channels is performed by a nearest neighbor interpolation (NNI).

The segmentation process occurs as follows: a digital color image $I[n, m]$ is separated in Red, Green and Blue channels, where each color channel is decomposed calculating their wavelets coefficients using Mallat's pyramid algorithm [3]. Then, using the biorthogonal 6.8 wavelet family, the original image is decomposed into four sub-bands. Three of these sub-bands, named LH, HL and $\mathrm{HH}$ represent the finest scale wavelet coefficient (detail images), while the subband LL corresponds to coarse level coefficients (approximation image), noted below as $D_{h}\left(2^{i}\right), D_{v}\left(2^{i}\right), D_{d}\left(2^{i}\right)$, and $A\left(2^{i}\right)$, respectively at given scale $2^{j}$, for $j=1,2$, ... $J$, where $J$ is the number of scales used in the Discrete Wavelet Transform (DWT) [4].

The DWT is represented as follows:

$W_{i}=\left|W_{i}\right| \exp \left(j * \theta_{i}\right)$, 
$\left|W_{i}\right|=\sqrt{\left|D_{h, i}\right|^{2}+\left|D_{v, i}\right|^{2}+\left|D_{d, i}\right|^{2}}$

where $\left|W_{i}\right|$ is the wavelet modulus on a chosen decomposition level $i$; $D_{h, i}, \quad D_{v, i}, \quad D_{d, i}$ are the horizontal, vertical and diagonal detail components on a level $i$, and the phase $\Theta_{i}$, is defined as follows:

$\theta_{i}=\left\{\begin{array}{llc}\alpha_{i} & \text { if } & D_{h, i}>0 \\ \pi-\alpha_{i} & \text { if } & D_{h, i}<0\end{array}\right.$,

$\alpha_{i}=\tan ^{-1}\left(D_{v, i} / D_{h, i}\right)$.

Consequently, $\mathrm{W}_{i}$ is considered as a new image for each color channel. The next step is the Fuzzy C-Means segmentation, where the segmented image corresponding to the red channel is interpolated with the segmented image corresponding to the green channel. This new image is obtained by applying a $N N I$. The NNI is repeated, taking the segmented image corresponding to the blue channel. The image that is obtained at the end of these interpolations is considered the output of the segmentation step.

By using the three color channels in the segmentation process, the extraction of clinically-relevant features is improved, thus making the classification more accurate, as compared when the original image is used to extract relevant features.

The color segmentation can be executed while the lesion segmentation is taking place. The color segmentation process is explained next.

\subsection{Color Segmentation}

Another variable that is used to diagnose skin cancer is the color content of the lesion. To detect what colors are present in the image, color segmentation is done by an Ensemble of Clusterers (EoCls). We decided to use EoCls because they are thought to overcome the limitations of single clustering algorithms by exploiting diversity in data processing. An EoCls can be obtained by using clustering algorithms on the same data or by using different values to the parameters of a single algorithm [5]. The EoCls employed to detect the RGB values of the colors that are present in a lesion is formed by three different algorithms: K-Means, Fuzzy C-Means and a Kohonen map. These algorithms run in parallel, each on its own thread, making the color extraction process faster. Each of the clusterers extracts the representative values of the partitions detected in the image being analyzed. Then, by averaging each channel representative, a global RGB value is obtained for each color. 


\subsection{Creation of Features Vector}

Texture analysis is one of the most important stages for a better classification because texture features provide special characteristics present in the image. Several authors have proposed methods to extract features of dermoscopy images $[6,7,8,9,10]$. However, those methods extract statistical properties and do not consider both local and global spatially correlated relationships among pixels. As opposed to the mentioned reports, we calculate the feature extraction using the GLCM method. The features vector includes: assymetry, area of the lesion, eccentricity, all the features in GLMC, and the color content of the lesion.

\subsection{Learning and Predicting by an Ensemble of Classifiers}

Classification is the task of learning a target function $f$ that maps the description of a certain set of instances to the values of a predefined attribute known as class. The input data for solving a problem of this kind is a collection of $N$ instances, which are characterized by a tuple $(X, y)$, where $X$ is a set of attributes and $y$ is the attribute that indicates the class label [11]. Classification has two main purposes: (i) descriptive modeling that explains the behavior between objects of different classes, and (ii) predictive modeling used for assigning a class label of an unknown instance.

For the problem of skin cancer detection, the classification task objective is to assign an input object $x_{\text {input }}$ to one of the binary outputs malignant tumor or benign tumor. Input $x_{\text {input }}$ possesses the set of features extracted during the lesion segmentation and color segmentation stages (see Section 3 for details).

Nonetheless, single classification algorithms do not always provide the most accurate predictions. To overcome this limitation, an ensemble of classifiers is proposed. Ensembles of classifiers are thought to outperform individual classifiers because they allow to filter out hypothesis that are not accurate due to a small training set; ensembles of classifiers help overcoming problem of local optima; different classifiers expand the universe of available target functions $f$ [12].

The ensemble of classifiers that we developed (named MAEoC since it is developed following the Multi-Agent paradigm) acts on two premises: (i) the performance of base classifiers and (ii) the communication of hits $(\mathrm{H})$ and failures (F) obtained by base classifiers. The design of MAEoC can be consulted in [13]. The MAEoC works according to the following algorithm:

Iteration $\mathrm{t}=0$

$m$ classifiers, $m>2$ are recruited and $m$ classifier agents are started.

Dataset $D$ containing features is broadcasted to classifier agent ${ }_{i}$, for all $i$, $i=1, \ldots m$. 
Classifier $_{i}$ performs a ten fold cross-validation. F-Measure $_{\mathrm{i}}$ is calculated. Classifier ${ }_{i}$, for all $i, i=1 \ldots m$, constructs two subsets. Subset $H_{i}$ contains objects correctly classified; subset $F_{i}$ contains objects incorrectly classified.

Iteration $\mathrm{t}=1$

Aggregated sets $A H$ and $A F$ are formed. $A H=\cup_{i} H_{i} ; \mathrm{AF}=\cup_{i} \mathrm{~F}_{i}$. classifier $_{m+1}$, is started, based on the highest F-Measure ${ }_{i}$ obtained at $t=0$.

Classifier $_{m+l}$ is trained with set $A F$. F-Measure $\mathrm{C}_{m+1}$ is obtained by ten fold cross validation on $A F$.

Classifiers $_{1, \ldots, m}$ are trained with set $A H$. F-Measures ${ }_{1, \ldots, m}$ are obtained by ten fold cross-validation on $A H$.

Iteration $\mathrm{t}=2$

Classifiers $_{1, \ldots, \mathrm{m}+1}$ are given weights according to their updated FMeasure at $t=1$. Weighted voting is used to reach a final conclusion.

The algorithms that form the ensemble of classifiers are: a Multilayer Perceptron (MLP) [14], a Naive Bayes classifier [15], a decision tree C4.5 [16], a K nearestneighbor [17], and a support vector machine [18].

Classification metrics are obtained to measure the performance of the ensemble. We compare the performance of the MAEoC with those of the individual classifiers that make it up. The MAEoC is also contrasted with classical aggregation methods such as Bagging [19], Boosting [20], and Stacking [21].

\subsubsection{Classification Metrics}

The following metrics are employed to evaluate the performance of classifiers: sensitivity, specificity, precision, recall, true positive rate, false positive rate, and the area under the ROC curve (AUC) and F-Measure.

Firstly, to determine how well the segmentation algorithm performs, it requires a ground truth (GT) image, which is determined by drawing manually the border around the lesion. Using a GT image, the exclusive disjunction (XOR) operation is calculated [22]. For dermoscopy images, sensitivity measures the proportion of actual lesion pixels that are correctly identified as such. Specificity measures the proportion of background skin pixels that are correctly identified. Generalizing:

- TP (true positive). Objects that are correctly classified as the object of interest.

- FP (false positive). Objects that are incorrectly identified as the object of interest. 
- TN (true negative). Objects that are correctly identified as not being the object of interest.

- FN (false negative). Objects that are incorrectly identified as not being the object of interest.

Sensitivity and specificity are given by:

$$
\begin{aligned}
& \text { sensitivity }=T P /(T P+T N) \\
& \text { specificity }=T N /(F P+T N)
\end{aligned}
$$

The precision of a classifier is the fraction of tuples that were correctly classified as positive from all the tuples that are actually positve. Precision is defined as follows:

$P=$ precision $=(T P) /(T P+F P)$.

Recall is the fraction of positive tuples that were correctly classified as positive:

$R=$ recall $=(T P) /(T P+F N)$.

We also apply the Receiver Operating Characteristic (ROC) analysis. Points of the ROC curve are obtained by sweeping the classification threshold from the most positive classification value to the most negative. A quantitative summary of the ROC curve is called the area under the ROC curve (AUC).

Classification is also quantified by the $F$-measure, defined as the weighted harmonic mean of its precision and recall:

$F=2 P R /(P+R)$.

The F-measure assumes values in the interval $[0,1]$. It is 0 when no relevant instances have been retrieved, and is 1 if all retrieved instances are relevant and all relevant instances have been retrieved. Experimental results are given in the following section.

\section{Experimental Results}

This section provides the results of determining the occurrence or not of skin cancer on 147 images of the ISIC repository. All of the images are stored as 24-bit color image in JPEG format. They have already been characterized with both, Ground Truth and the diagnosis given by the expert. Even though we do not contemplate the pre-processing of the images as part of the proposed method, occlusions and artifacts were removed in all the images by applying the DullRazon algorithm [23]. 
Figures 2 and 3 show the lesion segmentation process. In them, Figure $(c)$ illustrates the result of the segmentation after applying the W-FCM algorithm on figures $(a)$. When comparing the final result $(c)$ with figure $(b)$ (Ground Truth of lesion), the W-FCM displays higher precision and accuracy.

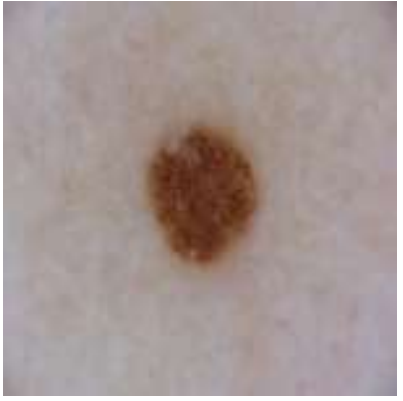

(a)

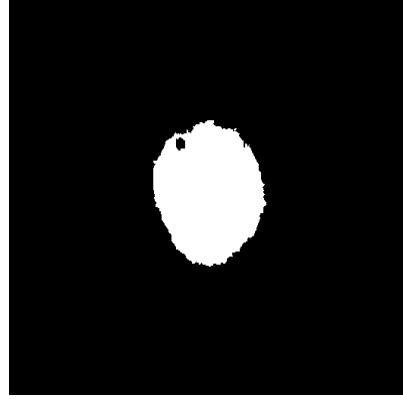

(b)

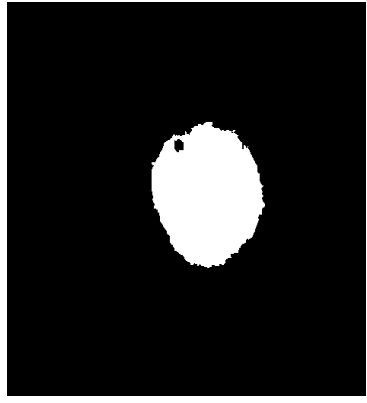

(c)

Figure 2

(a) Image ISIC_0000261 skin lesion benign accord to data set (b) Ground Truth as delineated by an expert (c) Segmentation with the W-FCM method. The following metrics are obtained: Precision = 0.99317, Sensitivity $=0.9998$, Specificity $=0.92388$, Accuracy $=0.99355$

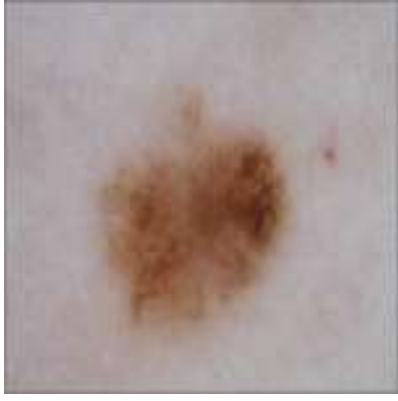

(a)

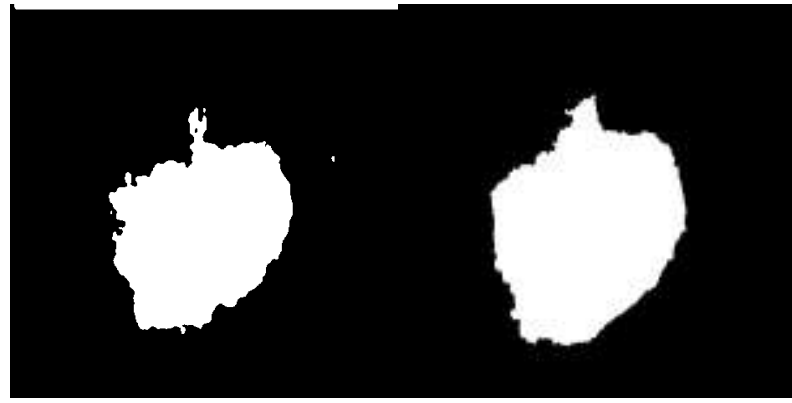

(b) (c)

Figure 3

(a) Image ISIC_0000054 skin lesion malign according to data set (b) Ground Truth (c) Segmentation with the W-FCM method. The following metrics are obtained: Precision $=0.94664$, Sensitivity $=$ 0.98260, Specificity $=0.78957$, Accuracy $=0.94239$ 
As for the color segmentation, we exemplify this stage with the following digital images. Figure 4(a) shows a digital image of dermoscopy taken form the ISIC repository. After applying a Kohonen Map to discover the most representative values, Figures 4(b) and 4(c) are obtained. For this particular image only two colors were discovered. The result of this stage is the obtainment of the RGB values of each of the segmented images. Needless to say that such values are added to the final features vector.

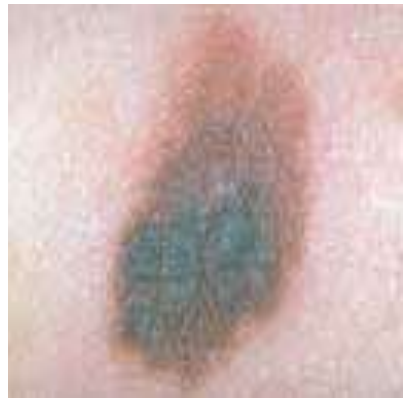

(a)

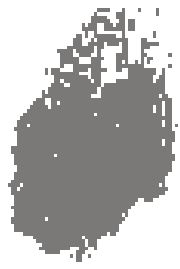

(b)

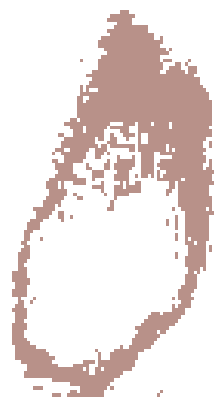

(c)

Figure 4

Illustration of the color segmentation stage. (a) Original digital image taken from ISIC. (b) Example of color segmentation of Figure 4(a) by using a Kohonen Map. (c) Second color found in Figure 4 (a) by using a Kohonen Map

Altogether, the vector of extracted features contains the assymmetry of the lesion, the eccentricity value, the area of the lesion, all the features of GLCM (i. e. autocorrelation, energy, entropy, dissimilarity), and the RGB values of the found colors, according to the ABCD guide. This complete vector of features is the actual input to the MAEoC.

The performances of both, the MAEoC and its constituting classifiers are presented in the following three tables. Metrics were obtained after running a tenfold cross validation.

Table 1 presents classification metrics when the vector consists in the following features: Colors quantity, texture features and morphology features. Table 2 presents the performance when, in addition to the features that were used to obtain Table 1, the RGB values of each found color are added to the vector of features. Finally, Table 3 presents the performance of the classifiers when the area of each found color is included in the features vector. 
Table 1

Classification results using number of colors, texture features and morphology features

\begin{tabular}{|l|c|c|c|c|}
\hline Classifier & Accuracy & ROC & Average Precision & $\begin{array}{c}\text { F- } \\
\text { Measure }\end{array}$ \\
\hline Multi Layer Perceptron & 0.631 & 0.513 & 0.628 & 0.630 \\
\hline Support Vector Machine & 0.77 & 0.5 & 0.594 & 0.671 \\
\hline Decision Trees & 0.708 & 0.408 & 0.624 & 0.657 \\
\hline Naive Bayes & 0.604 & 0.465 & 0.646 & 0.622 \\
\hline KNN; k =3 & 0.715 & 0.428 & 0.61 & 0.652 \\
\hline KNN; k =5 & 0.77 & 0.9 & 0.715 & 0.694 \\
\hline AdaBoost & 0.729 & 0.498 & 0.618 & 0.66 \\
\hline Bagging & 0.729 & 0.52 & 0.618 & 0.66 \\
\hline Stacking & 0.77 & 0.464 & 0.594 & 0.671 \\
\hline MAEoC & $\mathbf{0 . 8 8 8}$ & $\mathbf{0 . 7 8 9}$ & $\mathbf{0 . 9 0 3}$ & $\mathbf{0 . 8 7 5}$ \\
\hline
\end{tabular}

Table 2

Classification results using number of colors, texture features, morphology features, and RGB values obtained in the color segmentation phase

\begin{tabular}{|l|c|c|c|c|}
\hline Classifier & Accuracy & ROC & Average Precision & F-Measure \\
\hline Multi Layer Perceptron & 0.666 & 0.492 & 0.652 & 0.659 \\
\hline Support Vector Machine & 0.77 & 0.5 & 0.594 & 0.671 \\
\hline Decision Trees & 0.673 & 0.473 & 0.619 & 0.643 \\
\hline Naive Bayes & 0.611 & 0.444 & 0.634 & 0.622 \\
\hline KNN; k = & 0.729 & 0.488 & 0.638 & 0.669 \\
\hline KNN; k =5 & 0.77 & 0.441 & 0.712 & 0.683 \\
\hline AdaBoost & 0.729 & 0.405 & 0.618 & 0.66 \\
\hline Bagging & 0.75 & 0.508 & 0.639 & 0.672 \\
\hline Stacking & 0.77 & 0.464 & 0.594 & 0.671 \\
\hline MAEoC & $\mathbf{0 . 8 4}$ & $\mathbf{0 . 7 1 6}$ & $\mathbf{0 . 8 6 8}$ & $\mathbf{0 . 8 0 5}$ \\
\hline
\end{tabular}

Table 3

Classification results using number of colors, texture features, morphology features, RGB values obtained in the color segmentation phase, and the area of the lesion

\begin{tabular}{|l|c|c|c|c|}
\hline Classifier & Accuracy & ROC & Average Precision & F-Measure \\
\hline Multi Layer Perceptron & 0.68 & 0.484 & 0.681 & 0.681 \\
\hline Support Vector Machine & 0.77 & 0.5 & 0.594 & 0.67 \\
\hline Decision Trees & 0.631 & 0.428 & 0.611 & 0.621 \\
\hline Naive Bayes & 0.611 & 0.425 & 0.649 & 0.628 \\
\hline KNN; k =3 & 0.729 & 0.467 & 0.638 & 0.669 \\
\hline
\end{tabular}




\begin{tabular}{|l|c|c|c|c|}
\hline KNN; $\mathrm{k}=5$ & 0.756 & 0.471 & 0.674 & 0.686 \\
\hline AdaBoost & 0.701 & 0.449 & 0.581 & 0.636 \\
\hline Bagging & 0.736 & 0.517 & 0.588 & 0.654 \\
\hline Stacking & 0.77 & 0464 & 0.594 & 0.671 \\
\hline MAEoC & $\mathbf{0 . 8 4}$ & $\mathbf{0 . 6 6 8}$ & $\mathbf{0 . 8 6 8}$ & $\mathbf{0 . 8 0 5}$ \\
\hline
\end{tabular}

It can be noticed that the best metrics correspond, in these three cases, to MAEoC. In the following section, we present a comparison of how the MAEoC fares when comparing its performance with the related work presented in the literature.

However, it is worth noticing that the performance of the MAEoC decreases slighty when the number of features of the input vector increases. This effect can be seen on the accuracy values reported in Table $1(0.88)$ and Tables 2 and 3 (0.84). Also, the area under the ROC curve decreases from 0.789 in Table 1 , to 0.716 in Table 2 , and 0.668 in Table 3.

One possible explanation refers to the nature of the lesion under analysis. Since melanin is a substance determinant in the pigmentation of the skin, a malign melanoma changes the naturally occurring color of the skin, as well as its texture. In this sense, data such as the area of the lesion might as well be of no relevance. That is to say, the area of the lesion could be small or large and yet the effects on melanin are noticeable changes on color and texture. More experimentation is needed, though.

\section{Comparison with other Methods}

Computer Aided Diagnosis (CAD) systems for malignant melanoma have been developed rather recently. Although not all of the systems necessarily include the same processes, the following steps are common: image pre-processing, feature extraction, color interpretation, classification and lesion evaluation. A review of such systems is given in [24], and we selected five systems displaying the best performance. A summary is given in Table 4.

The classification methods that have been used in those five top-performers employ $\square$-Nearest Neighbor, Decision Trees, Support Vector Machines, Artificial Neural Network (ANN), Neuro-Fuzzy, Fuzzy C-Means, and Naive Bayes. The best results are obtained when hybrid techniques are employed. Even though the ensemble of classifiers that we developed is not strictly a hybrid system, it does benefit from using multiple classifiers for the detection of malignant lesions.

Moreover, these hybrid systems are trained with a large set of features extracted from the digital images. In the system we present, the features vector also displays a high dimensionality, although the quantity of images we process is not large (147 images). 
We consider that more details should have been given in those reports. For instance, the source of the images is not made explicit as opposed to the images we use, which are available to a broad community (the ISIC repository). Neither is it clear whether the images employed in those CAD systems were pre-processed in order to eliminate artifacts.

Table 4

Comparison of the proposed method with other approaches

\begin{tabular}{|c|c|c|c|c|c|}
\hline Autor & Dataset & $\begin{array}{c}\text { Pre } \\
\text { processing }\end{array}$ & $\begin{array}{l}\text { Feature } \\
\text { extraction } \\
\text { method }\end{array}$ & Classifier & $\begin{array}{c}\text { Detection } \\
\text { performance }\end{array}$ \\
\hline $\begin{array}{l}\text { (Sheha et } \\
\text { al) [25] }\end{array}$ & $\begin{array}{c}102 \\
\text { dermoscopy } \\
\text { Atlases }\end{array}$ & $\begin{array}{c}\text { Resizing } \\
\text { and Color } \\
\text { space } \\
\text { Transformat } \\
\text { ion }\end{array}$ & GLCM & $\begin{array}{l}\text { Multi- } \\
\text { Layer } \\
\text { Perceptro } \\
\text { n }\end{array}$ & $\begin{array}{l}\text { Accuracy } \\
=\% 92\end{array}$ \\
\hline $\begin{array}{c}\text { (KumarJai } \\
\text { n \& Jain) } \\
{[26]}\end{array}$ & $\begin{array}{c}\text { From } \\
\text { different } \\
\text { sources }\end{array}$ & $\begin{array}{c}\text { Image } \\
\text { contour } \\
\text { Tracing } \\
\text { Algorithm }\end{array}$ & $\begin{array}{c}\text { Discrete } \\
\text { Wavelet } \\
\text { Transform }\end{array}$ & $\begin{array}{c}\text { Clustering } \\
\& \\
\text { k-Nearest } \\
\text { Neighbors }\end{array}$ & $\begin{array}{c}\text { Accuracy }= \\
92 \% \\
\text { Accuracy }= \\
95 \%\end{array}$ \\
\hline $\begin{array}{c}\text { (Elgamal) } \\
{[27]}\end{array}$ & $\begin{array}{c}\text { From a } \\
\text { digital } \\
\text { camera with } \\
\text { dermoscope }\end{array}$ & $\begin{array}{c}\text { Gaussian - } \\
\text { Median } \\
\text { Filter }\end{array}$ & $\begin{array}{c}\text { Principal } \\
\text { Component } \\
\text { Analysis. } \\
\text { Discrete } \\
\text { Wavelet } \\
\text { Transform }\end{array}$ & $\begin{array}{c}\text { Artificial } \\
\text { Neural } \\
\text { Networks, } \\
\text { k-Nearest } \\
\text { Neighbors } \\
\text {. }\end{array}$ & $\begin{array}{c}\text { Accuracy }= \\
95 \% \\
\text { Accuracy }= \\
97.5 \%\end{array}$ \\
\hline $\begin{array}{c}\text { (Mengistu } \\
\text { ) [28] }\end{array}$ & $\begin{array}{c}\text { Dermquest/ } \\
\text { Dermnet }\end{array}$ & $\begin{array}{l}\text { Median } \\
\text { Filtering }\end{array}$ & $\begin{array}{c}\text { GLCM and } \\
\text { color } \\
\text { features }\end{array}$ & $\begin{array}{c}\text { Self } \\
\text { Organizin } \\
\text { g Maps } \\
\text { and Radial } \\
\text { Basis } \\
\text { Functions }\end{array}$ & $\begin{array}{c}\text { Accuracy }= \\
96.15 \%\end{array}$ \\
\hline $\begin{array}{l}\text { (Immagul } \\
\text { ate \& } \\
\text { Vijaya) } \\
\text { [29] }\end{array}$ & $\begin{array}{l}\text { Dermnet/ } \\
\text { Dermofit }\end{array}$ & $\begin{array}{l}\text { Image } \\
\text { resizing }\end{array}$ & $\begin{array}{l}\text { Color and } \\
\text { Texture } \\
\text { Features }\end{array}$ & $\begin{array}{l}\text { Support } \\
\text { Vector } \\
\text { Machine }\end{array}$ & $\begin{array}{c}\text { Accuracy }= \\
86 \%\end{array}$ \\
\hline $\begin{array}{l}\text { Proposed } \\
\text { System }\end{array}$ & $\begin{array}{l}\text { ISIC } \\
\text { repository }\end{array}$ & $\begin{array}{c}\text { Artifact } \\
\text { removal } \\
\text { with Razor } \\
\text { algorithm }\end{array}$ & $\begin{array}{c}\text { Fuzzy } \\
\text { Discrete } \\
\text { Wavelet } \\
\text { Transform }\end{array}$ & $\begin{array}{c}\text { Multi- } \\
\text { Agent } \\
\text { ensemble } \\
\text { of } \\
\text { Classifiers }\end{array}$ & $\begin{array}{c}\text { Accuracy }= \\
88 \%\end{array}$ \\
\hline
\end{tabular}




\section{Conclusions and Future Work}

One of the main problems to obtain a good performance regarding segmentation and classification in dermoscopy images refers to the proper selection of the features that characterize a skin lesion. To solve this problem, we propose a method consisting in the following stages: lesion segmentation, feature extraction, color extraction, and learning. An intelligent system was developed mirroring the mentioned steps.

Particularly, we have proposed the extraction of the following features: asymmetry, eccentricity, features of the well-known method called Grey Level Co-occurrence Matrix, and the color content of the lesion, for which an Ensemble of Clusterers is used. Nevertheless, the feature extraction is only one step in the automatic detection of skin cancer. The other major task for an intelligent system is learning from the combination of feature values that represent either a malignant tumor or a benign lesion. The learning and classification stage is performed by an ensemble of classifiers called MAEoC. As it is mentioned in the literature, ensembles of classifiers take advantage of the combined results of different classifiers. MAEoC is formed by a Multi-Layer Peceptron, a decision tree, a K nearest-neighbor, a Naïve - Bayes and a Support Vector Machine. The performance metrics indicate that MAEoC displays a better performance than single classifiers. However, aggregation methods such as stacking and bagging fare at least as well as the MAEoC.

One of the limitations of the results we present refers to the number of images that were analyzed. We are embarked in using a larger database than the 147 images that were processed in order to obtain the results given along the present article. Also, we are experimenting with more segmentation techniques, and algorithms that make adaptable both of the ensembles.

Another improvement is the addition of more relevant information to the features vector such as the ratio of the color area to the lesion area once the lesion has been separated from the original image. Regarding color extraction, we also envision the discovery of colors in a different color space than the RGB to include data such as hue and brightness.

\section{Acknowledgements}

The authors thank Universidad Autónoma del Estado de Hidalgo and SEP PRODEP for the financial support. The authors also want to thank Gonzalo Chávez Fragoso and Jaime Calderón for their time dedicated to program some modules of the system.

\section{References}

[1] H. Castillejos, V. Ponomaryov, L. Niño de Rivera and V. Golikov. Wavelet transform fuzzy algorithms for dermoscopic image segmentation. 
Computational and Mathematical Methods in Medicine. Vol. 2012, pp. 1121,2012

[2] H. Castillejos, V. Ponomaryov and R. Peralta-Fabi. Image segmentation in Wavelet Transform Space implemented on a DSP. Proceedings of the SPIE 8437. Real - time image and video processing. Vol. 8437, April 2012

[3] S. Mallat. A theory for multi-resolution signal decomposition: The Waveltet representation. IEEE Transactions on Pattern Analysis and Machine Intelligence. Vol. 11, No. 7, pp. 338-353, 1989

[4] V. Kravchenko, H. Meana and V. Ponomaryov. Adaptive digital processing of multidimensional signals with applications. Editorial FizMatLit, Kiev. 2009

[5] S. Mimaroglu and E. Erdil. An efficient and scalable family of algorithms for combining clusterings. Engineering Applications of Artificial Intelligence. Vol. 26, pp. 2525-2539, 2013

[6] I. Maglogiannis and D. Kosmopoulos. Computational vision systems for the detection of malignant melanoma classifiers using ROC curves. Oncology Reports. Vol. 15, pp. 1027-1032, 2006

[7] D. Ruiz, V. Berenguer, A. Soriano et al. A decision support system for the diagnosis of melanoma: a comparative approach. Expert Systems with Applications. Vol. 38, pp. 15217-15223, 2011

[8] T. Tanaka, S. Torii, I. Kabuta et al. Pattern classification of nevus with texture analysis. EEJ Transactions on Electrical and Electronic Engineering. Vol. 3, pp. 143-150, 2008

[9] C. Serrano and B. Acha. Pattern analysis of dermoscopic images based on Markov random fields. Pattern Recognition. Vol. 42, pp. 1052-1057, 2009

[10] M. Sadegui, M. Razmara, T. Lee et al. A novel method for detection of pigment networks in dermoscopic images using graphs. Computerized Medical Images and Graphics. Vol. 35, pp. 137-143, 2011

[11] Pang-Ning Tang, Michael Steinbach and Vipin Kumar. Introduction to Data Mining. Addison - Wesley, 2006

[12] Michael Wozniak, Manuel Grana and Emilio Corchado. A survey of multiple classifier systems as hybrid systems. Information Fusion. Vol. 16, pp. 3-17, 2014

[13] Jaime Calderón, Omar López-Ortega and Félix Castro-Espinoza. A multiagent ensemble of classifiers. Advances in Artificial Intelligence and Soft Computing: $14^{\text {th }}$ Mexican International Conference on Artificial Intelligence, MICAI 2015, Cuernavaca, Morelos, Mexico, October 25-31, 2015, Proceedings, Part I, pp. 499-508, 2015 
[14] Christopher M. Bishop. Neural networks for pattern recognition. Oxford University Press, 1995

[15] Nir Friedman, Dan Geiger and Moises Goldszmidt. Bayesian networks classifiers. Machine Learning. Vol. 29, No. 2-3, pp. 131-163, 1997

[16] J. Ross Quinlan. C4:5: Programs for machine learning. Elsevier, 2014

[17] T. Cover and P. Hart. Nearest neighbor pattern classification. IEEE Transactions on Information Theory. Vol. 13, No. 1, pp. 21-27, 1967

[18] Corrina Cortes and Vladimir Vapnik. Support-vector networks. Machine learning. Vol. 20, No. 3, pp. 273-297, 1995

[19] Leo Breiman. Bagging predictors. Machine Learning. Vol. 24, No. 2, pp. 123-140, 1996

[20] Yoav Freund and Robert E. Schapire. A decision - theoretic generalization of on-line learning and an application to Boosting. Journal of Computer and System Sciences. Vol. 55, pp. 119-139, 1997

[21] Leo Breiman. Stacked regressions. Machine Learning. Vol. 24, No. 1, pp. 49-64, 1996

[22] N. Lachiche and P. A. Flach. Improving accuracy and cost of two-class and multi-class probabilistic classifiers using ROC curves. In Proceedings of the ICLM, Vol. 2003, pp. 1027-1032, 2006

[23] T. Lee, V. Ng, R. Gallagher, A. Coldman and D. McLean. DullRazor: A software approach to hair removal from images. Computers in Biology and Medicine. Vol. 27, pp. 533-543, 1997

[24] M. A. Arasi, , E. S. A. El-Dahshan, E. S. M. El-Horbaty, and A. B. M. Salem. Malignant Melanoma Detection Based on Machine Learning Techniques: A Survey. Egyptian Computer Science Journal. Vol. 40, No. 3, pp. 1-10, September 2016

[25] M. Sheha, M. Mabrouk and A. Sharawy. Automatic detection of melanoma skin cancer using texture analysis. International Journal of Computer Applications. Vol. 42, No. 20, pp. 22-26, 2012

[26] Y. Kumar Jain and M. Jain. Comparison between different classification methods with application to skin cancer. International Journal of Computer Applications. Vol. 53, No. 11, pp. 18-24, 2012

[27] M. Elgamal. Automatic Skin Cancer Images Classification. International Journal of Advanced Computer Science and applications. Vol. 4, No. 3, pp. 287-294, 2013

[28] A. D. Mengistu. Computer Vision for Skin Cancer Diagnosis and Recognition using RBF and SOM. International Journal of Image Processing. Vol. 9, pp. 311-319, 2015 
[29] I. Immagulate and M. S. Vijaya. Categorization of Non-Melanoma Skin Lesion Diseases Using Support Vector Machine and Its Variants. International Journal of Medical Imaging. Vol. 3, No. 2, pp. 34-40, 2015 